\title{
Complications of bariatric surgery: presentation and emergency management - a review
}

\author{
SJW MONKHOUSE, JDT MORGAN, SA NORTON
}

\author{
Department of Surgery, Southmead Hospital, Bristol, UK
}

ABSTRACT

INTRODUCTION The prevalence of obesity surgery is increasing rapidly in the UK as demand rises. Consequently, general surgeons on-call may be faced with the complications of such surgery and need to have an understanding about how to manage them, at least initially. Obesity surgery is mainly offered in tertiary centres but patients may present with problems to their local district hospital. This review summarises the main complications that may be encountered.

MATERIALS AND METHODS A full literature search was carried out looking at articles published in the last 10 years. Keywords for search purposes included bariatric, surgery, complications, emergency and management.

CONCLUSIONS Complications of bariatric surgery have been extensively written about but never in a format that is designed to aid the on-call surgeon. The intricate details and rare complications have been excluded to concentrate on those symptoms and signs that are likely to be encountered by the emergency team.

\section{KEYWORDS}

Obesity - Bariatric surgery - Complications - Emergency management

\section{CORRESPONDENCE TO}

Simon JW Monkhouse, Department of Surgery, Southmead Hospital, Bristol BS10 5NB, UK

E: sjwmonkhouse@hotmail.com

Obesity is a world-wide epidemic ${ }^{1}$ and is associated with multiple serious co-morbidities, both physical and psychological. Over the last decade, there has been an exponential rise in the number of bariatric procedures being offered, ${ }^{2}$ as these have consistently been shown to be the only way to achieve sustainable weight loss and improvement in comorbidities, particularly type II diabetes and hypertension. There are many different surgical operations available to achieve weight loss; the choice of surgery depends on a number of factors, not least the experience of the surgeon and the patient's individual requirements. The predominant operation performed in the UK is the laparoscopic adjustable gastric band (LAGB), which is a purely restrictive procedure. Other procedures incorporate a malabsorptive element and include the Roux-en-Y gastric bypass (RYGB) and the biliopancreatic diversion (with or without duodenal switch; BPD, BPD/DS; Fig. 1A). Other operations include sleeve gastrectomy (Fig. 1B) which can be used as a definitive procedure or as a bridge to further by-pass surgery, and the largely historic vertical banded gastroplasty. All surgery carries some degree of risk and the decision to operate is made after a careful balance of the risks versus the benefits. This particular group of patients often have limited physiological reserves and this, together with their physical size, may make the surgery high-risk. Early detection and appropriate management of complications is crucial to prevent long-term morbidity and mortality.

This review highlights the main complications that the general surgeon on-call may encounter as an emergency and illustrates the appropriate management strategies.

A full literature search was carried out using PubMED and the Cochrane Library. Relevant international articles published in the last 10 years were assessed. Keywords for search purposes included bariatric, surgery, complications, emergency and management.

\section{Band-related complications}

\section{Dysphagia/band slippage}

Immediate postoperative dysphagia is seen in some patients following LAGB. This is usually due to excessive perigastric fat resulting in a tightly fitting band or to postoperative oedema. Complete dysphagia, even for saliva, may take up to 10 days to resolve. Postoperative intravenous steroids and a strict nil-by-mouth regimen appear to increase the resolution rate for the oedema and thus hasten recovery. These patients are often in-patients until postoperative dysphagia resolves and so it is late dysphagia that more commonly 


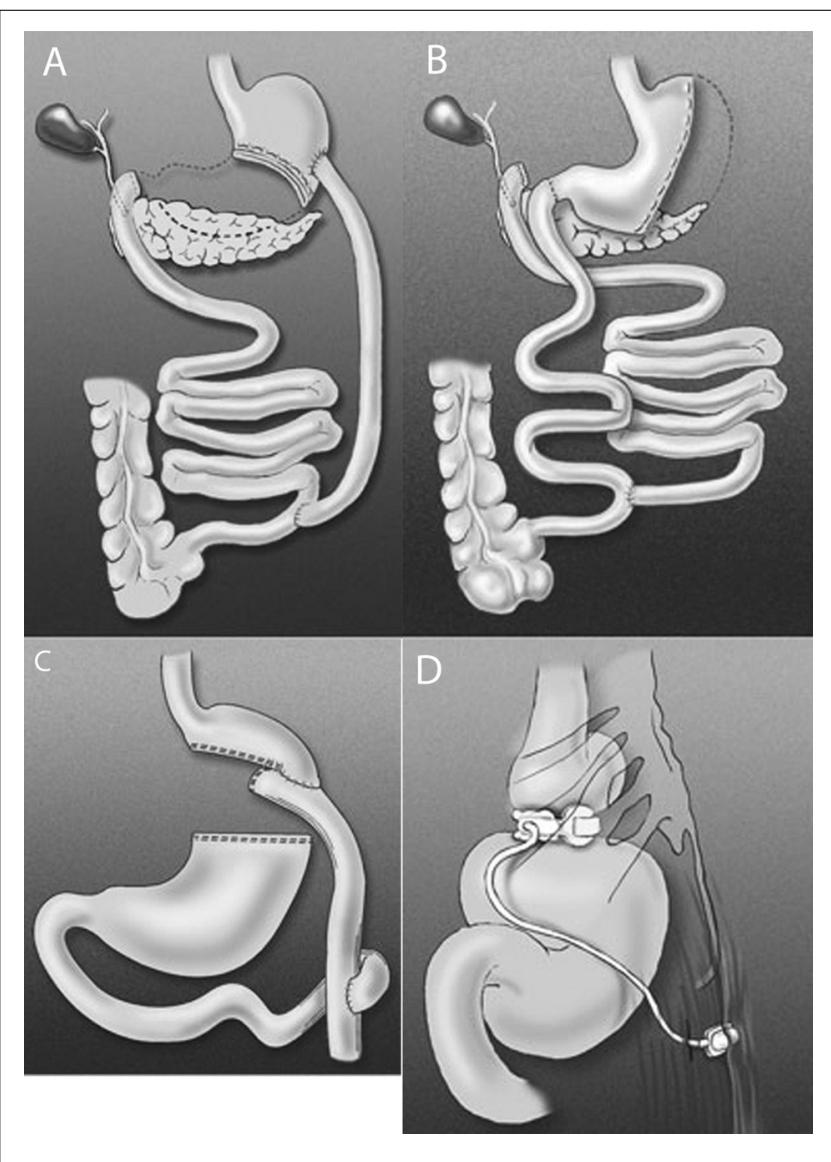

Figure 1 The main types of bariatric surgery available. Reprinted with permission of the American Society for Metabolic and Bariatric Surgery (C) 2008, all rights reserved. A, biliopancreatic diversion (BPD); B, biliopancreatic diversion with duodenal switch (BPD/DS); C, Roux-en-Y gastric bypass (RYGB); D, laparoscopic adjustable gastric band (LAGB).

presents to the acute surgical take. Symptoms are often progressive and result in an inability to swallow saliva in extreme cases. Dysphagia may be due to a food bolus obstruction or, of more concern, may represent a band slippage. An acute band slippage (incidence of 1-3\%) is defined as a 'cephalic prolapse of the stomach's inferior portion with consequent caudal slippage of the band'. ${ }^{3}$ This may cause complete gastric outlet obstruction. A history of excessive vomiting prior to onset of symptoms is often noted.

Emergency management necessitates immediate band deflation (see below) whilst investigations are being ordered. A two-view barium swallow is the investigation of choice (Figs 2 and 3 ) and is diagnostic. However, a plain radiograph (Fig. 4) is often sufficient to identify the horizontal (or occasionally vertical) lie of a band after gastric herniation as opposed to its normal $45^{\circ}$ oblique position in the anteroposterior view. It is important to ensure that the radiograph captures the band as it is often subcostal in position and may be missed by a standard abdominal X-ray (Fig. 4).

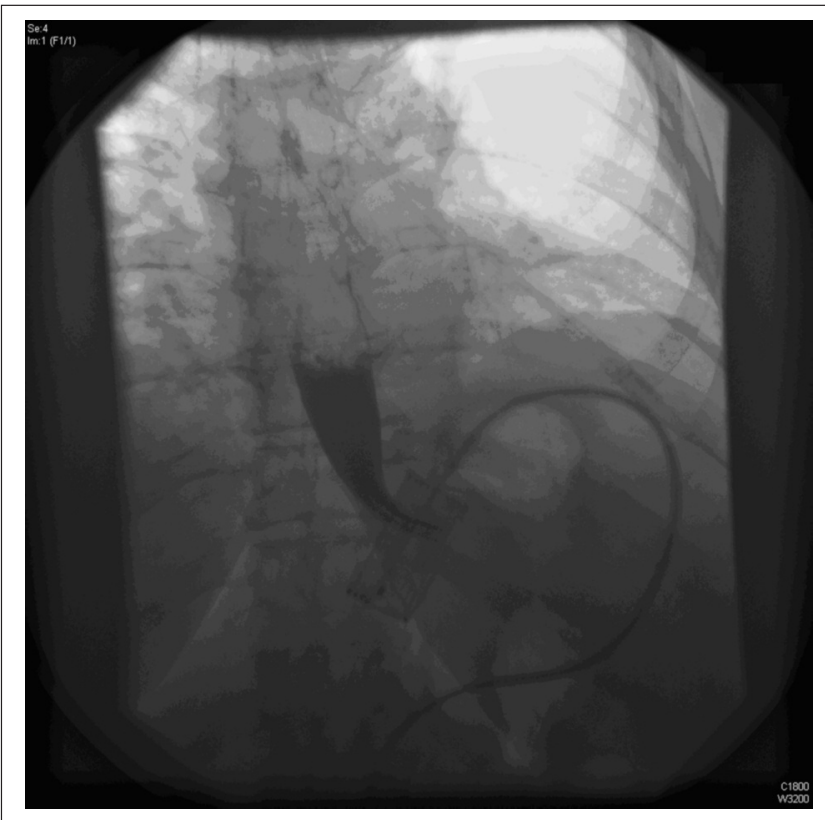

Figure 2 Good band position on barium swallow. Note $45^{\circ}$ of band, no pouch dilatation and flow of contrast through the band.

If there is no evidence of band slippage then band deflation followed by a review of the patient's eating habits, to ensure appropriate food choices and careful chewing, may be all that is necessary. Occasionally, a cautious endoscopy (after band deflation) may be needed to clear a large food bolus and intravenous fluids are required whilst oedema

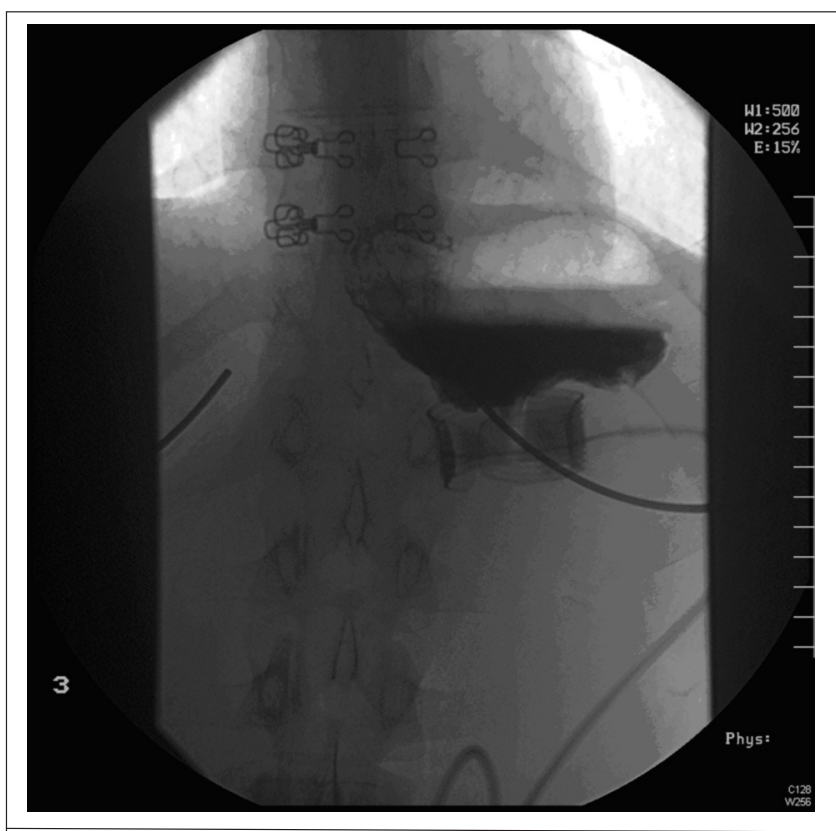

Figure 3 An acute gastric band slip. Note the horizontal lie of the band and the dilated pouch above the band. Contrast does not flow through the band. 


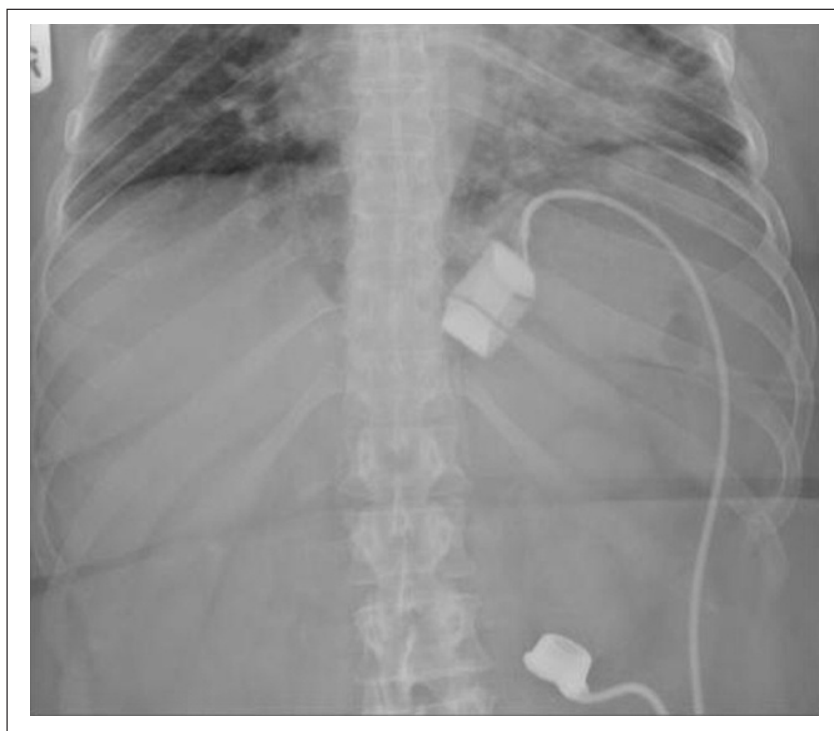

Figure 4 A plain emergency $\mathrm{X}$-ray showing a gastric band in situ (good band position).

settles. These should all be performed by the emergency surgical team.

If band slippage is radiologically confirmed, immediate deflation of the band via the subcutaneous port is required (if not already done). If this does not produce a rapid improvement in symptoms, then re-operation is necessary to avoid the risk of gastric ischaemia. ${ }^{3}$ It is important to note that pain may be minimal even in cases of incipient gastric ischaemia and more subtle signs, such as tachycardia, may alert the surgeon to the need for intervention. A raised lactate level and acidosis may indicate gastric necrosis and the need for urgent surgery if there has been delay in presentation or treatment. In the absence of a specialist bariatric team, laparoscopy and band removal can be performed by a competent laparoscopic general surgeon. There is often a fibrous capsule around the band which requires dissection before band removal and stomach wall may have been sutured around the band for fixation. All surgeons should familiarise themselves with the locking mechanisms of the common bands in use so that emergency removal can be facilitated. Fulminant gastric necrosis should be managed by appropriate gastric resection, after band removal.

If dysphagia resolves following band deflation, surgical intervention can usually be delayed until the patient has been seen by the specialist bariatric team. Band salvage may be possible, otherwise removal with potential for re-banding or other forms of bariatric surgery may be performed.

\section{BAND DEFLATION}

Band deflation is a simple procedure that all general surgeons need to be aware of, as timely band deflation can prevent the onset of gastric ischaemia. The port may be difficult to palpate but the patient is generally aware of its precise location. The most common positions are in the left upper quadrant, anterior to, or just below the lower sternum or just lateral to the umbilicus. ${ }^{4}$ The depth of the port also varies between centres. Some are immediately subcutaneous and unsecured; ${ }^{4}$ others are fixed by means of sutures to the rectus sheath. If the port is not easily palpable, $\mathrm{X}$-ray guidance may be required.

Band deflation should be performed under strict aseptic conditions to avoid introducing infection into the band system. A non-coring needle (e.g. Huber needle) is used if possible (Fig. 5). The access port should be held fixed between the thumb and index finger of the non-dominant hand, the smooth dome identified and the needle smoothly inserted until it touches the metallic base of the port. The fluid should be aspirated to dryness. Most of the patients will know how much fluid is currently in their band and this can be used as a guide for the emergency surgeon. Immediate resolution of symptoms often follows and a timely referral to a bariatric surgeon should be made.

\section{Severe reflux-like symptoms}

Patients may present to the acute take with severe epigastric, burning pain or chest pain. Such symptoms may be secondary
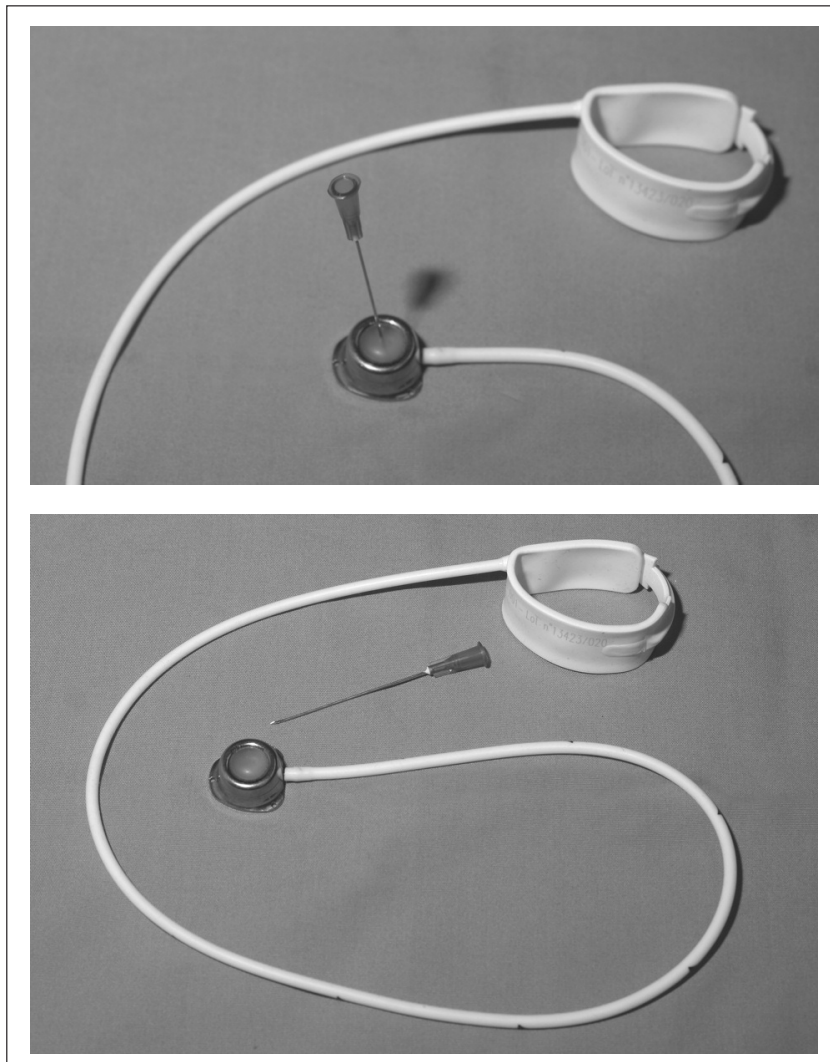

Figure 5 The subcutaneous inflation/deflation port and gastric band. Note the smooth dome which should be palpable. A Huber needle used for inflation/deflation is shown. 
to stasis in a poorly functioning oesophagus, which can become increasingly dilated (pseudo-achalasia) due to a high or over-tight band. Initial management is immediate band deflation, as described above. All patients should have high-dose proton-pump inhibitors prescribed, if not already part of their normal prescription. If symptoms settle, a referral to the bariatric team should be made for consideration of gradual re-filling of the band over many months. Failure to resolve symptoms may necessitate removal of the band and conversion to a sleeve gastrectomy, RYGB or BPD to maintain weight loss; ${ }^{2}$ this would be not be undertaken by the acute general surgeons.

\section{Band erosion}

This is relatively rare (incidence $4 \%$ or less) ${ }^{5}$ but can be a cause of bleeding, pain or infection. Erosion of the band through the stomach wall is a slow process, such that it rarely results in perforation and subsequent peritonitis. The eroded band is exposed to gastric bacterial flora and the tubing can subsequently become infected. One of the first signs of gastric erosion may, in fact, be evidence of recurrent infection around the access port. This should be a warning sign to the emergency surgeon of a more sinister, underlying problem. Antibiotics may be required and an urgent referral made to the specialist centre. There has been a reported case of an eroded band migrating and causing small bowel obstruction. ${ }^{6}$ An emergency laparotomy may be necessary in this situation. An alternative presentation is with weight re-gain as the band loses its restrictive effect. An eroded band is diagnosed on contrast studies, endoscopy or both and can be treated by laparoscopic or endoscopic removal depending on degree of erosion. In fact, in the absence of septic complications, band removal may be delayed for many months in the hope of near complete intra-gastric migration occurring to facilitate an endoscopic removal. This is best done in an specialist bariatric unit with experience of revisional surgery and where alternative weight loss surgery can be considered if necessary. ${ }^{7}$

\section{Bypass-related complications}

\section{Anastomotic leak}

An anastomotic leak is a serious, life-threatening problem and can be difficult to diagnose due to the patient's size. The incidence of leaks following RYGB is $1-5.6 \% .^{8}$ Most present with non-specific signs of sepsis (tachycardia, leukocytosis, fever) within the first 10 postoperative days and it is common for a morbidly obese patient to have peritonitis with a clinically soft abdomen. ${ }^{2}$ Radiological investigations are difficult as the patient may not fit into a conventional computed tomography (CT) scanner and the scans do not have a high sensitivity for leaks. A large case series of 63 patients with 68 leaks following RYGB showed that 44\% were not detectable by CT scanning. ${ }^{8,9}$ A gastrograffin swallow and subsequent abdominal $\mathrm{X}$-ray is more sensitive and can demonstrate extravasation of contrast. However, both CT scans and contrast studies suffer from a significant falsenegative rate. Any patient with tachycardia and sepsis within 10 days of bypass surgery should, therefore, be considered for re-laparoscopy. These patients are usually still inpatients and thus surgery can be performed by the original bariatric team. However, if the patient has had an early discharge, they may present to the emergency on-call team. Analgesia, antibiotics, aggressive fluid resuscitation and urgent transfer back to the bariatric unit is usually optimum management. If symptoms are severe and the patient is deteriorating clinically, a laparoscopic washout and drain placement may be a temporising measure until definitive bariatric team management occurs. ${ }^{8}$ This can be performed by the general on-call surgeons. In the stable patient who is suitable for transfer, it is sometimes possible to stent leaks endoscopically with good results, ${ }^{10}$ thus avoiding high-risk surgery; this is a decision best made by the original team. Percutaneous radiological drainage of localised, contained leaks is also possible with some degree of success. ${ }^{8}$ These should be carried out in the bariatric unit with input from specialist radiologists and endoscopists.

\section{Anastomotic stricture}

Strictures can follow any gastrointestinal anastomosis. Incidence following RYGB is reported at 3-11\% depending on whether the procedure is performed open or laparoscopically. ${ }^{11,21}$ (The stricture rate seems to be higher in the laparoscopic group.) This may be more a reflection of the difference between hand-sewn and stapled anastomoses, rather than the operative approach. Causes of stricture include technical error, ischaemia, sub-clinical leak, tension or delayed fibrosis secondary to ulceration. ${ }^{12}$ Patients present with dysphagia, nausea and/or bowel obstruction, typically between 3 weeks and 3 months after surgery. ${ }^{19}$ Diagnosis is made on history and confirmed by endoscopy. An anastomosis of less than $10 \mathrm{~mm}$ in diameter requires endoscopic balloon dilation and a single dilation to $15-18 \mathrm{~mm}$ is often sufficient to resolve symptoms. Serial dilatations may be required for late strictures where there is a well-established fibrotic response. ${ }^{12,20}$ Perforation remains a real risk (incidence of up to $2.1 \%$ ), ${ }^{9}$ particularly within 4 weeks of operation. Endoscopy should ideally be performed in the specialist bariatric unit and supportive initial management is all that is required from the emergency on-call surgeon. Minimising the risk factors which contribute to ulceration can help to prevent re-stenosis. These include smoking cessation, avoidance of NSAIDs and the routine postoperative use of proton-pump inhibitors. ${ }^{9}$ For resistant, symptomatic stenoses, semi-elective revisional surgery is the only real treatment option. 


\section{Internal hernias}

These are a major cause of morbidity and mortality in the postoperative obese patient. In many malabsorptive procedures, there are up to three potential internal hernia spaces. The most common site of herniation is the so-called Peterson defect which is the space posterior to the Roux limb. Other sites are mesenteric defects. Internal hernias appear to be much less frequent after open surgery than following laparoscopic surgery. Possible reasons for this include less postoperative adhesions after laparoscopic surgery and surgeons failing to close the mesenteric defects after laparoscopic surgery due to extra technical effort and time required. ${ }^{2}$ Internal hernias can result in intestinal obstruction, ischaemia or both; these usually present with intermittent abdominal pain and, less commonly, vomiting. Vomiting is not always a feature due to the reconfigured anatomy. The pain may be related to food and is often partially relieved by lying prone or 'on all fours'. ${ }^{2}$ The emergency surgeon must be mindful of these hernias in any postoperative patient with severe abdominal pain. Blood tests are often unrewarding ${ }^{17}$ as are CT scans. In one series, $20 \%$ of CT scans were reported as normal in patients who subsequently underwent surgery for incarcerated internal hernias. ${ }^{20}$ The serum amylase level may be raised due to obstruction of the biliopancreatic limb which may result in the misdiagnosis of pancreatitis. ${ }^{2}$ The on-call surgeon should operate if signs of intestinal necrosis are present (pain, acidosis, lactate rise). Resection of necrotic segments together with closure of the mesenteric defects is appropriate. With the Peterson defect, the herniated bowel should be reduced and sutured in its correct position. ${ }^{2}$ Ideally, this should be performed by the original bariatric team, but such surgery should clearly not be delayed if bowel ischaemia is suspected.

\section{All operations}

\section{Pulmonary embolism}

Postoperative pulmonary embolism is the major cause of death in this group. ${ }^{13}$ The incidence of pulmonary embolism is $1-2 \%$ in this population, with up to $50 \%$ of these being fatal. Measures taken to reduce this risk include pre-operative low molecular weight heparin, intra-operative pneumatic calf compression and early mobilisation postoperatively. Clinical signs in this group can be non-specific and are difficult to distinguish from other postoperative respiratory conditions such as atelectasis and pneumonia. Chest $\mathrm{X}$ rays are often unhelpful as is physical examination. CT angiography of the thorax is the gold standard for those in whom clinical suspicion is high. However, in the super obese group (body mass index $>50 \mathrm{~kg} / \mathrm{m}^{2}$ ), imaging may be impossible due to limitations of scanner size. In this group, prophylactic anticoagulation is advisable, ${ }^{2}$ despite the increased risk of bleeding from staple lines or suture holes. Ren et al. ${ }^{14}$ reported a clinically significant haemorrhage from anastomoses in $10 \%$ of postoperative patients receiving formal anticoagulation.

\section{Gallstones}

Cholelithiasis is common following obesity surgery (incidence of up to $30 \%)^{9}$ and may be precipitated by rapid weight loss. ${ }^{15}$ These patients present to the surgical take with all the signs and symptoms of gallstone disease. They are at risk of cholecystitis, ascending cholangitis and pancreatitis, as with other patient groups. Their management can be slightly different, however. Imaging may be difficult due to sub-optimal views. Endoscopic retrograde cholangiopancreatography (ERCP) may be difficult or impossible in the presence of altered anatomy. Laparoscopic cholecystectomy may also be technically challenging due to postoperative adhesions. Acute management should focus on symptom relief and treatment of biliary sepsis (i.e. antibiotics, analgesia and fluids). Definitive cholecystectomy may be best performed by the bariatric surgeon if there is concern about altered anatomy.

Prophylactic cholecystectomy during bariatric surgery remains controversial. Some groups advocate removal of all gallbladders at bariatric surgery; others recommend removing only those in which gallstones are demonstrated intra-operatively or when performing bypass surgery where weight loss is more rapid and gallstone complications appear more frequent. Others prefer to leave all gallbladders in situ at the original operation and deal with complications if they arise. ${ }^{16}$

\section{Port-site hernias}

Port-site hernias from laparoscopic procedures may present with painful swellings and are usually the result of failure to close the fascial defect at time of surgery. If bowel is involved, obstruction with vomiting may occur. These can be managed by urgent surgery by the on-call team necessitating reduction of the hernial contents, bowel resection if necessary, and definitive closure of the defect (with or without mesh depending on clinical context). Port-site surgery can either be done open or laparoscopically depending on experience.

\section{Gastrointestinal bleeding}

Immediate postoperative bleeding is usually from staple lines or from poor haemostasis at the time of surgery. Reoperation is often indicated and will be carried out by the bariatric in-patient team. From $72 \mathrm{~h}$ onwards, patients with severe bleeding may present to the acute take. Erosions and ulceration can occur in the gastric remnant and at anastomoses. Supportive care including blood transfusion, correction 


\section{Table 1 Summary of management protocols}

\begin{tabular}{|c|c|c|c|}
\hline Symptom & Possible diagnosis & Emergency on-call management options & Specialist bariatric management options \\
\hline $\begin{array}{l}\text { Complete } \\
\text { dysphagia }\end{array}$ & $\begin{array}{l}\text { Food bolus, } \\
\text { band slip }\end{array}$ & $\begin{array}{l}\text { Intravenous fluids; immediate band deflation; } \\
\text { X-ray and/or contrast swallow; possible } \\
\text { endoscopy; laparoscopic removal of band } \\
\text { if no rapid relief with deflation; urgent } \\
\text { referral to SBU if possible }\end{array}$ & $\begin{array}{l}\text { Band revision or replacement; conversion to } \\
\text { alternative bariatric procedure }\end{array}$ \\
\hline $\begin{array}{l}\text { Sepsis, } \\
\text { abdominal } \\
\text { pain }\end{array}$ & $\begin{array}{l}\text { Anastomotic } \\
\text { leak }\end{array}$ & $\begin{array}{l}\text { Supportive; laparoscopic washout/drain; } \\
\text { percutaneous CT-guided drainage; } \\
\text { referral to SBU }\end{array}$ & $\begin{array}{l}\text { Endoscopic stent, re-operation with } \\
\text { resection and re-anastomosis }\end{array}$ \\
\hline $\begin{array}{l}\text { Recurrent } \\
\text { port-site } \\
\text { infection }\end{array}$ & $\begin{array}{l}\text { Band } \\
\text { infection/ } \\
\text { erosion }\end{array}$ & Antibiotics, fluids. Referral to SBU & $\begin{array}{l}\text { Removal of band. Conversion to alternative } \\
\text { procedure }\end{array}$ \\
\hline $\begin{array}{l}\text { Vomiting, } \\
\text { nausea, } \\
\text { pain }\end{array}$ & $\begin{array}{l}\text { Stricture, } \\
\text { internal } \\
\text { hernia }\end{array}$ & $\begin{array}{l}\text { Supportive, nasogastric tube; } \\
\text { emergency laparotomy and resection; } \\
\text { referral to SBU }\end{array}$ & $\begin{array}{l}\text { Balloon dilatation; emergency laparotomy; } \\
\text { revisional bariatric surgery }\end{array}$ \\
\hline Reflux & $\begin{array}{l}\text { Pseudo- } \\
\text { achalasia }\end{array}$ & Band deflation; PPI; referral to SBU & $\begin{array}{l}\text { Cautious re-fills; band removal and } \\
\text { conversion to alternative procedure }\end{array}$ \\
\hline Bleeding & $\begin{array}{l}\text { Erosions, } \\
\text { ulcers }\end{array}$ & $\begin{array}{l}\text { Transfusion; correct coagulopathy; } \\
\text { high-dose PPI; endoscopy, laparotomy }\end{array}$ & $\begin{array}{l}\text { Specialist endoscopy; consideration of } \\
\text { laparotomy }\end{array}$ \\
\hline $\begin{array}{l}\text { Wound } \\
\text { cellulitis }\end{array}$ & $\begin{array}{l}\text { Infection, } \\
\text { impending } \\
\text { dehiscence }\end{array}$ & $\begin{array}{l}\text { Antibiotics, wound swabs; incision } \\
\text { and drainage of collections }\end{array}$ & Out-patient bariatric follow-up \\
\hline
\end{tabular}

SBU, specialist bariatric unit; PPI, proton-pump inhibitor.

of clotting abnormalities and acid suppression is often all that is required. Partial bowel obstruction can occasionally result due to the presence of a luminal clot. ${ }^{12}$ Endoscopy can be attempted at this stage but may be difficult due to altered anatomy. Refractory bleeding from the remnant stomach or other sites inaccessible by endoscopy may necessitate re-operation but this should certainly be managed by an experienced bariatric team if time allows. Recurrent, intractable gastric bleeding may require a gastrectomy due to limitations in access. ${ }^{22}$

\section{Wound problems}

Patients may present with wound cellulitis and sepsis. The most frequent organisms are Staphylococcus aureus, Enterococcus spp. and $\alpha$-haemolytic Streptococcus spp.
Patients who suffer a wound infection are at high risk of developing an incisional hernia. ${ }^{23}$ and early, aggressive treatment with antibiotic therapy can help prevent fascial dehiscence (incidence of $1 \%) .{ }^{9}$ Recurrent access port-site infection is concerning, as indicated above, and can represent an infected or eroded band. This should be referred to the bariatric unit after antibiotic therapy.

There are many other complications associated with bariatric surgery but these do not usually present to the emergency on-call surgeon. These include mineral or vitamin deficiencies secondary to malabsorption and dumping syndrome. These tend to be managed with dietary modifications and support by the outreach bariatric service in the community. Table 1 summarises the management options for the complications of bariatric surgery. 


\section{Conclusions}

Bariatric surgery is becoming more prevalent; as a result, complications are also becoming more wide-spread. Emergency surgeons need to understand the various types of surgery that have been performed so that they can understand the complications and manage them appropriately. Signs are often subtle and non-specific and the surgeon must be mindful of postoperative problems. The simple act of being able to deflate a gastric band in an emergency may be life-saving. Ideally, attempts should be made to contact the specialist bariatric team whenever such patients present to ensure appropriate management decisions; however, this is not always possible. Emergency management must always be followed by referral back to the dedicated bariatric team to continue treatment and provide appropriate support to the patient, but also to ensure comprehensive audit in this evolving specialty.

\section{References}

1. Prentice AM. The emerging epidemic of obesity in developing countries. Int J Epidemiol 2006; 35: 93-9.

2. Herron DM, Bloomberg R. Complications of bariatric surgery. Minerva Chir 2006; 61: 125-39.

3. Singhal R, Kitchen M, Ndrika S, Hunt K, Bridgwater S, Super P. The 'Birmingham stitch' - avoiding slippage in laparoscopic gastric banding. Obes Surg 2008; 18: 359-63.

4. Arvind N, Bates SE, Morgan JD, Hewin DF, Frering VM, Norton SA. Fixation of the access-port is not required. Obes Surg 2007; 17: 577-80.

5. Biagini J, Karam L. Ten years experience with laparoscopic adjustable gastric banding. Obes Surg 2008; 18: 573-7.

6. Egbeare D, Myer A, Lawrance R. Small bowel obstruction secondary to intragastric erosion and migration of a gastric band. J Gastrointest Surg 2008; 12: 983-4.

7. Abu-Abeid S, Bar Zohar D, Sagie B, Klausner J. Treatment of intra-gastric band migration following laparoscopic banding: safety and feasibility of simultaneous laparoscopic band removal and replacement. Obes Surg 2005; 15: 849-52.

8. Gonzalez R, Sarr MG, Smith CD, Baghai M, Kendrick M, Szomstein S et al. Diagnosis and contemporary management of anastomotic leaks after gastric bypass for obesity. J Am Coll Surg 2007; 204: 47-55.

9. Lee C, Kelly J, Wassef W. Complications of bariatric surgery. Curr Opin Gastroenterol 2007; 23: 636-43.

10. Salinas A, Baptista A, Santiago E, Antor M, Salinas H. Self-expandable metal stents to treat gastric leaks. Surg Obes Relat Dis 2006; 2: 570-2.

11. Peifer KJ, Shiels AJ, Azar R, Rivera RE, Eagon JC, Jonnalagadda S. Successful endoscopic management of gastrojejunal anastomotic stricture following Rouxen-Y gastric bypass. Gastrointest Endosc 2007; 66: 253-5.

12. Elder KA, Wolfe BM. Bariatric surgery: a review of procedures and outcomes. Gastroenterology 2007; 132: 2253-71.

13. Sapala JA, Wood MH, SchuhKneckt MP, Sapal MA. Fatal pulmonary embolism after bariatric operations for morbid obesity: a 24 year retrospective analysis. Obes Surg 2003; 13: 819-25.

14. Ren CJ, Patterson E, Gagner M. Early results of laparoscopic biliopancreatic diversion with duodenal switch; a case series of 40 consecutive patients. Obes Surg 2000; 10: 514-23.

15. Fakhry SM, Herbst CA, Buckwalter JA. Cholecystectomy in morbidly obese patients. Am Surg 1987; 53: 26-8.

16. Guadalajara H, Sanz Baro R, Pascual I, Blesa I, Rotundo GS, Lopez JM et al. Is prophylactic cholecystectomy useful in obese patients undergoing gastric bypass? Obes Surg 2006; 16: 883-5.

17. Comeau E, Gagner M, Inabnet WB, Herron DM, Quinn TM, Pomp A. Symptomatic internal hernias after laparoscopic bariatric surgery. Surg Endosc 2005; 19: 34-9.

18. Higa KD, Ho T, Boone KB. Internal hernias after laparoscopic Roux- en-Y gastric bypass: incidence, treatment and prevention. Obes Surg 2003; $13: 350-4$.

19. Nguyen NT, Rivers R, Wolfe BM. Factors associated with operative outcomes in laparoscopic gastric bypass. J Am Coll Surg 2003; 197:548-55.

20. Schirmer B, Erenoglu C, Miller A. Flexible endoscopy in the management of patients undergoing Roux-en-Y gastric bypass. Obes Surg 2002; 12: 634-8.

21. Nguyen NT, Goldman C, Rosenquist J, Arango A, Cole CJ, Lee SJ et al. Laparoscopic versus open gastric bypass: a randomised study of outcomes, quality of life, and costs. Ann Surg 2001; 234: 279-91.

22. Braley SC, Nguyen NT, Wolfe BM. Late gastrointestinal haemorrhage after gastric bypass. Obes Surg 2002; 12: 404-7.

23. Christou NV, Jarand J, Sylvestre JL, Mclean APH. Analysis of the incidence and risk factors for wound infections in open bariatric surgery. Obes Surg 2004; 14: $16-22$. 